\title{
Climate change: understanding anthropogenic contributions and responses
}

\author{
Donald R. Nelson
}

Published online: 4 May 2010

(C) Springer Science+Business Media, LLC 2010

Climate change has emerged as the primary global challenge in the 21 st century. Even the attainment of the Millennium Development Goals has been subsumed by climate change, inasmuch as climate limits the ability to reach the goals. It is only natural therefore, that solutions to the problem, indeed even the way that the "problem" is framed are numerous. The idea of climate change is captured in questions of national-level attribution, or human security and justice, or may also be viewed as another aspect of Nature that we will learn to control through technology. Regardless of the way that the idea of climate change is understood, it does not detract from the rapid physical change documented in ecosystems around the globe. This special issue brings together a set of authors that address the idea of climate change through a range of perspectives that include vulnerability and adaptation, energy use, economic opportunities, and environmental governance. Together they provide a broad perspective of the complexities of responding to climate change and provide direction for thinking about impacts of climate change and our abilities to adequately respond to current and future challenges.

Although adaptation to dynamic environments is part of the human legacy, it hasn't always been marked by success nor have the benefits been equally distributed across society. Currently there is concern that societies previously adapted to variable climates will not have the capacities to adequately respond to the greater variation within a changing climate. McLeman (2010) explores changing adaptive capacities as a result of shifts in the demographic composition of a population. The author engages climate change through the concept of vulnerability, which is characterized by population attributes. Acknowledging that there is physical change

D. R. Nelson (ه)

Department of Anthropology, University of Georgia, 250A Baldwin Hall,

Jackson St., Athens, GA 30602, USA

e-mail: dnelson@uga.edu

D. R. Nelson

Tyndall Centre for Climate Change Research, University of East Anglia, Norwich, UK 
occurring, the research explores how technology, culture, social organization and demographic shifts, all reflect to some degree an evolving relationship with the physical environment and consequent levels of vulnerability. The highly detailed study provides the reader with a grounded sense of complex change processes in natural and human systems and how these changes influence vulnerabilities within the population. In addition, the author provides a typology of population change and vulnerability to a changing climate. This typology draws attention to the ways that vulnerabilities and adaptive capacities reflect population and demographic change.

Liddle and Lung (2010) explore the issue of carbon emissions in developed countries. The problem of climate change is approached as one in which human activities contribute to changing greenhouse gas concentrations. They conduct a STIRPAT analysis for 17 countries using observations from 1960 to 2005, at 5 year intervals. The analysis recognizes the importance of age-structural change in terms of per capita energy consumption and thus disaggregates population data into four age cohorts. The authors also employ improved variables to capture energy intensity. The analysis provides insights into how demographic composition influences energy use and thus carbon emissions. These first two manuscripts explore different aspects of climate change, and they both highlight the importance of understanding the demographic composition of study populations. A community, town, or nation may persist through time and may, as in McLeman's example, maintain a similar population level for some time. But the people, their individual and population level characteristics change. By disaggregating the populations in question, these studies enhance our understanding of how population attributes contribute to consumption patterns, consequent environmental impacts and potential vulnerabilities to a changing climate.

Researchers and policy makers recognize the influence of controlling parameters on the range of decisions that individuals choose from. Labor and commodity markets, credit opportunities, and reliance on variable environmental resources all influence the choices that individuals make in their daily lives. The aggregate results of individual actions have the potential to affect larger vulnerabilities and the development trajectories of regions and nations. From this perspective, Barbieri et al. (2010) contribute to our understanding of regional patterns of climate changerelated migration. They frame the problem of climate change in terms of altered economic opportunities. The authors explore the vulnerability of the dryland agricultural sector in Northeast Brazil and model the influence that a changing climate may have on long-established migration patterns. They employ a model based on demographic, technological, and economic scenarios to project future migration scenarios. These outputs are combined with two of the IPCC emission scenarios to identify potential changes in economic scenarios and migration patterns. The modeling highlights the uncertainty of future impacts, in light of the differences between emissions scenarios. Beyond this, however, is recognition of large-scale population shifts that may result from a changing climate and the need for public policy to address long-term change.

The ability to adapt entails more than technology or specific programmatic interventions, or even the capacity to project future scenarios. Adaptation to climate change must incorporate the linkages and relationships between individuals, society, 
and the environment, and stress response flexibility. The final manuscript in this special issue addresses the need for improved governance structures in order to respond adequately and equitably to climate challenges. Similar to the McLeman article, Mustelin et al. (2010) use the concept of vulnerability. They apply vulnerability is a starting point for exploring ways in which to promote adaptation strategies, and address climate change as a problem of environmental governance. In light of the lack of detailed climate change scenarios, the authors focus on how to identify and manage no-regret strategies that will reduce vulnerability to climate change. The authors evaluate physical changes in coastal Zanzibar and compare these with local perceptions of change as part of the process to identify these strategies. Resolution of current environmental problems through engagement with the wider community is recognized as a proxy for the ability to adequately respond to future change.

Thank you to all of the authors for their fine work, and to the reviewers who were an integral part of the process. I am also grateful to Lori Hunter for all of the assistance that she provided throughout the development of this issue.

\section{References}

Barbieri, A. F., Domingues, E., Queiroz, B. L., Ruiz, R. M., Rigotti, J. I., Carvalho, J. A. M., et al. (2010). Climate change and population migration in Brazil's Northeast: Scenarios for 2025-2050. Population and Environment. doi:10.1007/s11111-010-0105-1.

Liddle, B., \& Lung, S. (2010). Age-structure, urbanization, and climate change in developed countries: Revisiting STIRPAT for disaggregated population and consumption-related environmental impacts. Population and Environment. doi:10.1007/s11111-010-0101-5.

McLeman, R. A. (2010). Impacts of population change on vulnerability and the capacity to adapt to climate change and variability: A typology based on lessons from a hard country. Population and Environment. doi:10.1007/s11111-009-0087-z.

Mustelin, J., Klein, R. G., Asseid, B., Sitari, T., Khamis, M., Mzee, A., et al. (2010). Understanding current and future vulnerability in coastal settings: Community perceptions and preferences for adaptation in Zanzibar, Tanzania. Population and Environment. doi:10.1007/s11111-010-0107-z. 